\title{
Thermal analysis and thermographic measurements of piezoelectric transformers
}

\author{
by K. Tomalczyk and B. Wiecek
}

Institute of Electronics - Technical University of Lodz, Lodz, Poland

\begin{abstract}
This paper deals with the problem of thermal effects in piezoelectric transformers. With the development of piezoelectric technology, these high efficiency transformers are soon going to be used in a wide range of electronic equipment. With the rated power of piezoelectric transformers getting higher, despite their high efficiency (above 90\%), thermal effects caused by energy loss are becoming to be a great concern due to small size of the transformer. The paper discusses the results of thermal behaviour investigations of a ring-shaped piezoelectric transformer for step-down applications. The analysis consisted of two parts: finite element modelling and laboratory tests including thermal camera measurements.
\end{abstract}

\section{Introduction}

Piezoelectric transformers (PTs) are electrical energy transducers, whose working principle is based on mechanical resonance coupling of very high quality factor $(Q>1000)[1]$. Being much smaller, lighter, safer and more efficient, they constitute an interesting alternative to traditional magnetic-core transformers of comparable power. In optimal working conditions PTs reach up to $98 \%$ efficiency, which is unattainable for any standard transformer. The low power PTs (1-2W) for step-up applications have already been introduced to mass production and are currently a standard component of LCD monitors [5]. Much larger application scale is going to be achieved with the forthcoming introduction of PTs to general purpose AC/DC adaptors, present almost everywhere in consumer electronics. To fit this particular application a PT must be more powerful (at least $10 \mathrm{~W}$ of rated power) and suited for step-down operation. A prototype ring-shaped multi-layer PT fulfilling the above conditions has been developed by Danish piezoceramics specialist Noliac [8]. The mentioned PT operates at relatively high frequency range (approx. $420-470 \mathrm{kHz}$ ), where it should be able to transform up to $10 \mathrm{~W}$ with more than $90 \%$ efficiency [3]. The results of experimental verification of these declared values are presented in the second part of the paper.

The laboratory tests were preceded with a finite element analysis performed in ANSYS [9] modelling environment. The considered piezoelectric transformer structure was modelled as a multi-layer ring (3D with axial symmetry). To determine the main resonant modes of the transformer a modal analysis was performed first. Through the dynamic analysis performed for a set of near-resonance frequencies, the spatial distributions of maximum stress, displacement and electric field were obtained. For the modelling of energy loss, appropriate damping factors were introduced.

The main aim of the finite element analysis was to determine the distribution of energy loss within the piezoelectric material in order to find the source of heat generation in a working transformer. The resulting temperature distribution is then computed on the basis of volumetric heat generation rate introduced to the model.

The work presented is a part of a preliminary research related to the development process of piezoelectric transformer based power converters. The 
results presented in this paper will create a background for proper thermal management in the design of electrical equipment with piezoelectric transformers.

\section{Finite element analysis of piezoelectric transformers}

One of the important issues of PT design is its thermal behaviour in some particular working conditions. Despite its high efficiency when operating close to the optimal resonance frequency, even relatively small amount of energy loss can provoke a considerable thermal effect due to very high power density within the transfomer (up to $50 \mathrm{~W} / \mathrm{cm}^{3}$ for the ring-shaped PT [3]). In a typical application circuit, the output voltage is controlled through the variation of working frequency, which is directly related to the transformation ratio around the resonance. The change in frequency influences the efficiency, hence it should also have a thermal effect on the PT [4].

For the finite element analysis in the ANSYS environment, the ring-shaped $(12 \times 4 \mathrm{~mm})$ PT geometry was defined (figure 1). The piezoelectric material properties were introduced on the basis of material data for PZT-4 [6], one of the piezoelectrics suitable for high power applications. The input signal was assumed to be sinusoidal with a $40 \mathrm{~V}$ amplitude value.

As mentioned before, the piezoelectric transformers are able to operate efficiently only at some particular resonant frequencies. Therefore, a modal analysis was performed to determine these frequencies. A ring-shaped PT normally operates in its thickness mode, with the resonance frequency corrresponding to a standing wave across its smallest dimension ( $4 \mathrm{~mm}$ in this case). This resonant mode was chosen for further investigation from the set of frequencies obtained from the modal analysis.

For the chosen working frequency (about $464 \mathrm{kHz}$ ) a dynamic analysis was performed to determine the spatial distribution of stress and voltage (electric field) within the investigated PT structure. Due to the lack of complex theory describing the energy loss mechanism in a vibrating piezoelectric material, the built-in ANSYS damping factors were arbitrarily set to appropriate values, assuring at least qualititive modelling of the real behaviour of analysed PT structure. Figures 2 and 3 show the distributions of voltage and vertical stress for two opposite time points of the vibration period - the maximum positive and negative shape deviations from the equilibrium state.

The main difficulty of the analysis was to find a relation between mechanical and thermal domains. As the mechanism of energy losses in piezoelectric material is not yet discovered, we assumed an approximative approach that the heat generation rate is proportional to the relative displacement of a given point of the PT structure. The total energy loss within the PT was assumed to be $0.5 \mathrm{~W}$, resulting from a given power of $10 \mathrm{~W}$ with the worst-case efficiency $95 \%$ [4]. At the outer walls of the PT we assumed typical linear convection cooling conditions (alpha coefficient $=5 \mathrm{~W} / \mathrm{m}^{2} \mathrm{~K}$, external temperature $=300 \mathrm{~K}$ ). The comparison of obtained temperature and vibration amplitude distributions is presented in figure 4 .

\section{Laboratory efficiency tests and thermographic measurements}

For a proper efficiency measurement a precisely formed voltage sine wave should be input to the transformer. The transformer's output should be loaded with an adequate resistance, its value being calculated on the basis of assumed output voltage and power. In the case considered, the load resistance was fixed to $8.8 \Omega$, which corresponds to $12 \mathrm{~V}$ DC output voltage (after rectifying and filtering) at the power level of $10 \mathrm{~W}$. For this load value, at the resonance frequency (approx. 


\section{http://dx.doi.org/10.21611/qirt.2006.093}

$420 \mathrm{kHz}$ ) the input impedance of the transformer reaches its minimum of about $60 \Omega$, which was a reason to use a current source instead of a voltage one at the input. The test circuit diagram is shown in figure 5 .

A sine wave generator drives the input of an amplifier forcing a current flow through the input electrodes of the transformer. The current waveform $\left(l_{\text {in }}\right)$ applied to the transformer's input impedance results in an input AC voltage $\left(U_{\text {in }}\right)$ of a maximum amplitude reaching the level of $300 \mathrm{~V}$. As the pure sinusoidal input signal is central to the precision of the efficiency measurement, an adequately high DC bias voltage must be provided to avoid clipping. The inductor $L$ bypasses the transformer's input for the DC. To force 10 watts of real power into a $60 \Omega$ load, the input AC current must have the RMS value of about $400 \mathrm{~mA}$, that is approx. 1.1A peak-to-peak. As our test circuit is still in development, at the time of the experiment we were able to reach no more than 500mA peak-to-peak of undistorted current. This limits the maximum input power to approx. $2 \mathrm{~W}$, assuming that voltage and current are inphase, which is true only in a resonance condition. In practice, at the resonance frequency of $420 \mathrm{kHz}$, the peak attainable power was $1.35 \mathrm{~W}$.

The most effective technique would be the simultaneous measurement of all the values of interest $\left(U_{i n}, l_{i n}, U_{i n}, l_{i n}\right)$, which would allow us to calculate directly both the input and the output powers needed to evaluate the transformer's efficiency. At the frequencies of about $500 \mathrm{kHz}$, the current can be measured only by means of special laboratory current probes connected to the oscilloscope via dedicated amplifiers. Two such probes were used for $I_{\text {in }}$ and $I_{\text {out }}$. Two voltage probes for $U_{\text {in }}$ and $U_{\text {out }}$ could not be used in this particular case, as in contrary to the current probes they are not insulated from the test circuit. The common ground node of the oscilloscope short-circuits the primary and secondary side of the transformer, which has influence on its internal capacitance values and hampers the proper operation. Finally, a voltage probe was used only on the input. The input power was calculated by multiplying the voltage and current waveforms, while the output power calculation was performed on the basis of only one measured waveform (current) and known value of the load resistance (figure 6).

The first series of measurements was performed with an earlier version of the test circuit current source, capable to reach no more than $240 \mathrm{~mA}$ peak-to-peak. It resulted in correspondingly lower input power values. Table 1 presents the efficiency results obtained at a fixed output power rate $(0.4 \mathrm{~W})$ maintained across the entire frequency range of interest $(420-470 \mathrm{kHz})$ :

Table 1. Efficiency test results (part 1)

\begin{tabular}{|c|c|c|c|c|c|c|}
\hline $\mathbf{f ~ ( k H z )}$ & $\mathbf{4 2 0}$ & $\mathbf{4 3 0}$ & $\mathbf{4 4 0}$ & $\mathbf{4 5 0}$ & $\mathbf{4 6 0}$ & $\mathbf{4 7 0}$ \\
\hline Pin (W) & 0.43 & 0.42 & 0.41 & 0.44 & 0.44 & 0.47 \\
\hline Pout (W) & 0.40 & 0.40 & 0.40 & 0.40 & 0.40 & 0.40 \\
\hline $\mathbf{n}(\%)$ & 93 & 95 & 98 & 91 & 91 & 85 \\
\hline
\end{tabular}

Having the current source output capability boosted to $500 \mathrm{~mA}$, we were able to perform additional measurements at higher power rates $(0.8 \mathrm{~W}$ and $1.2 \mathrm{~W})$. They were done for comparison purposes at $420 \mathrm{kHz}$ (table 2).

Table 2. Efficiency test results (part 2)

\begin{tabular}{|c|c|c|}
\hline $\mathbf{f ~ ( k H z )}$ & 420 & 420 \\
\hline Pin (W) & 0.87 & 1.32 \\
\hline Pout (W) & $\mathbf{0 . 7 9}$ & $\mathbf{1 . 1 9}$ \\
\hline $\boldsymbol{\eta}(\%)$ & 91 & 90 \\
\hline
\end{tabular}


For higher frequencies, where the transformer's input impedance level increases, the $500 \mathrm{~mA}_{\mathrm{pp}}$ forced current resulted in much higher input power levels (up to $8 \mathrm{~W}$ ). In such conditions however, a considerable temperature rise followed by equally noticeable fall in efficiency was noticed. Figure 7 shows an example thermal image of the transformer at $440 \mathrm{kHz}$, with its maximum temperature exceeding $70^{\circ} \mathrm{C}$. Further power and efficiency measurements were abandoned to avoid a permanent damage to the transformer.

\section{Conclusion}

A qualititive mechanical and thermal finite element analysis of a new ringshaped piezoelectric transformer structure was perfomed. As the theory which would join these two classes of phenomena together for an exhaustive description of the piezoelectric material behaviour, is not yet developed, the results of our analysis are based on some approximative assumptions and cannot be treated as data for engineering calculations in a new design development process. They are however a good starting point for further quantitive analysis, provided that the PT finite element models will be fine-tuned by comparison with the laboratory test results, e.g. thermal camera measurements.

As a main result of the research, the ability of piezoelectric transformers to reach exceptionally high efficiency levels was proved. It was however noticed, that even at over $90 \%$ efficiency, the power losses are high enough, in relation to the volume of the transformer, to provoke a considerable temperature rise having negative influence on efficiency. Moreover, the differences between temperature distributions obtained experimentally and from finite element modelling make further investigations necessary to find a proper approach to the problem. Besides temperature, other conditions having effect on efficiency (fixing/placement technique, vibration damping) will be considered. An adequate cooling system for the piezoelectric transformer will be developed.

\section{REFERENCES}

[1] C.A. Rosen. Ceramic Transformers and Filters, Proc. Electronic Comp. Symp., 1956, 205-211.

[2] J.A. Oliver, R. Prieto, M. Sanz, J.A. Cobos and J. Uceda. 1D Modeling of Multilayer piezoelectric transformers. Universidad Politécnica de Madrid.

[3] T. Bove, W. Wolny, E. Ringgaard and K. Breboel. New Type of Piezoelectric Transformer With Very High Power Density. Ferroperm Piezoceramics A/S

[4] Development of the Demonstrators. TRAMST project report - D4.1 - Alcatel

[5] Ultracompact LCD Backlight Inverters. Linear Technology Application Note 81, 09.1999

[6] Morgan Electroceramics catalogue www.morgan-electroceramics.com

[7] K. Tomalczyk, M. Chalczynski and B. Wiecek. Thermomechanical analysis of piezoelectric transformers. $14^{\text {th }}$ International Conference on Thermal Engineering and Thermogrammetry THERMO 2005. Budapest, Hungary, 2005

[8] www.noliac.com

[9] www.ansys.com 
http://dx.doi.org/10.21611/qirt.2006.093

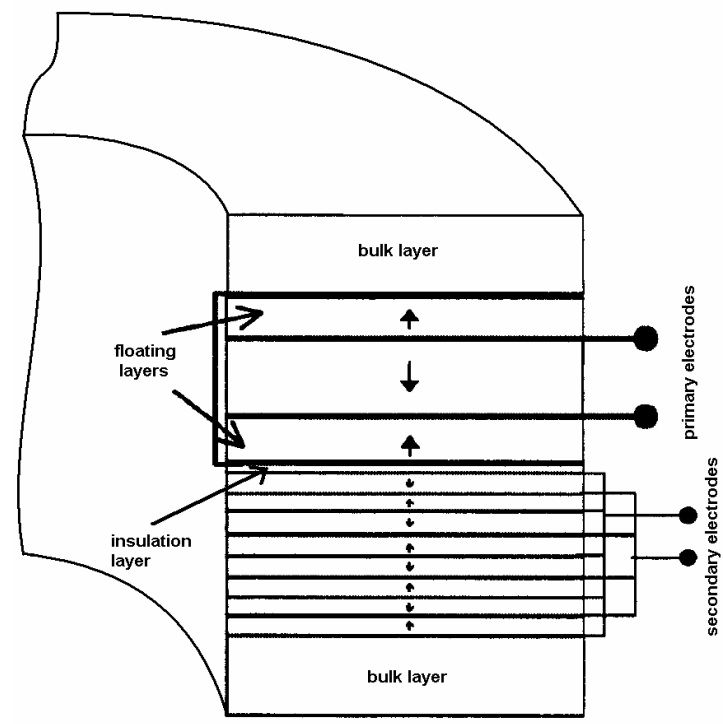

Fig. 1. Cross-section of the ring-shaped PT [2]. The arrows show polarization direction.
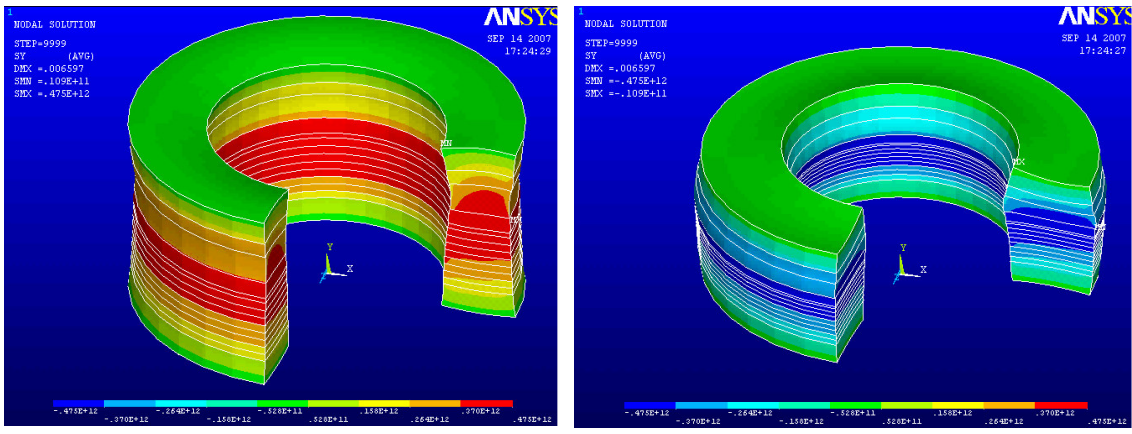

Fig.2. Example results of dynamic analysis - distributions of vertical stress in a ringshaped PT in the extreme points of the vibration period. The colour scale expresses stress in pascals. 

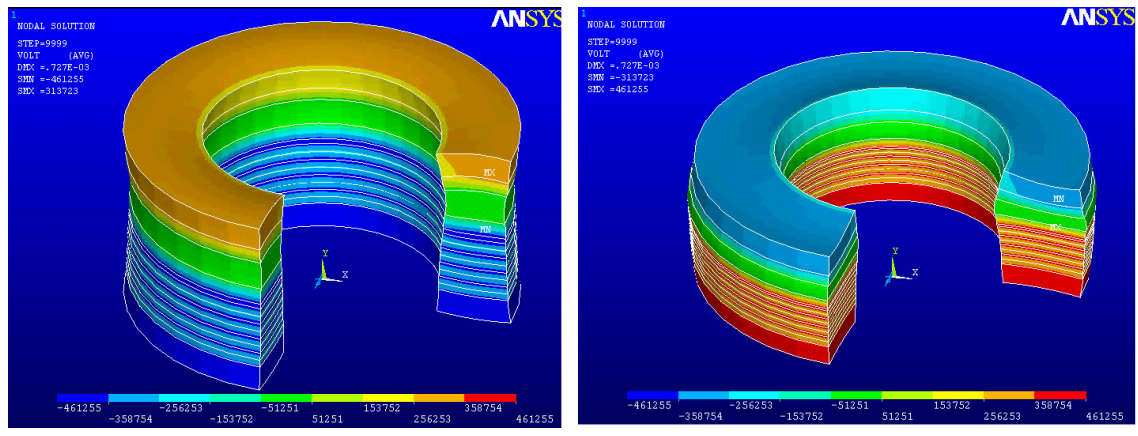

Fig. 3. Example results of dynamic analysis - voltage distributions in a ring-shaped $P T$ in the extreme points of the vibration period. The colour scale expresses voltage in volts.
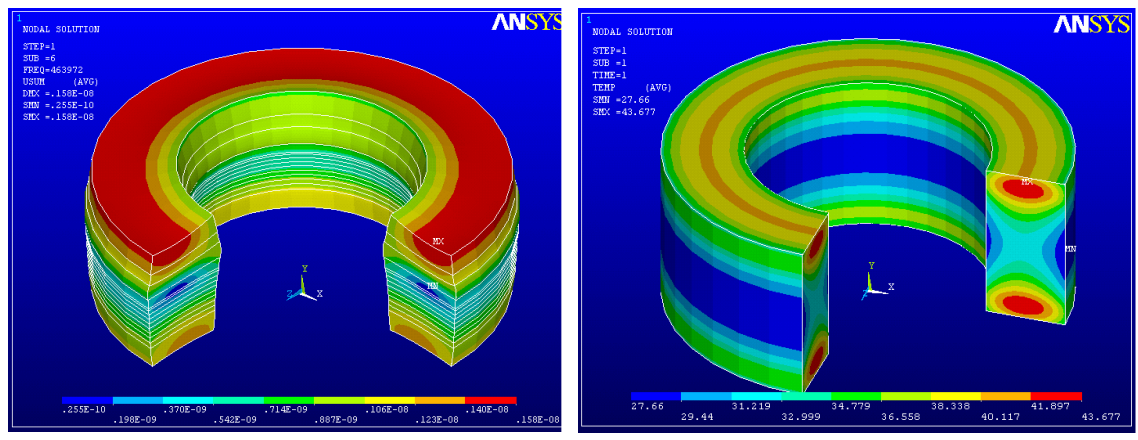

Fig. 4. Vibration amplitude (left) and temperature distribution (right) for the the ringshaped PT working in its main resonant mode. Temperature is expressed in Celsius degrees. 
http://dx.doi.org/10.21611/qirt.2006.093

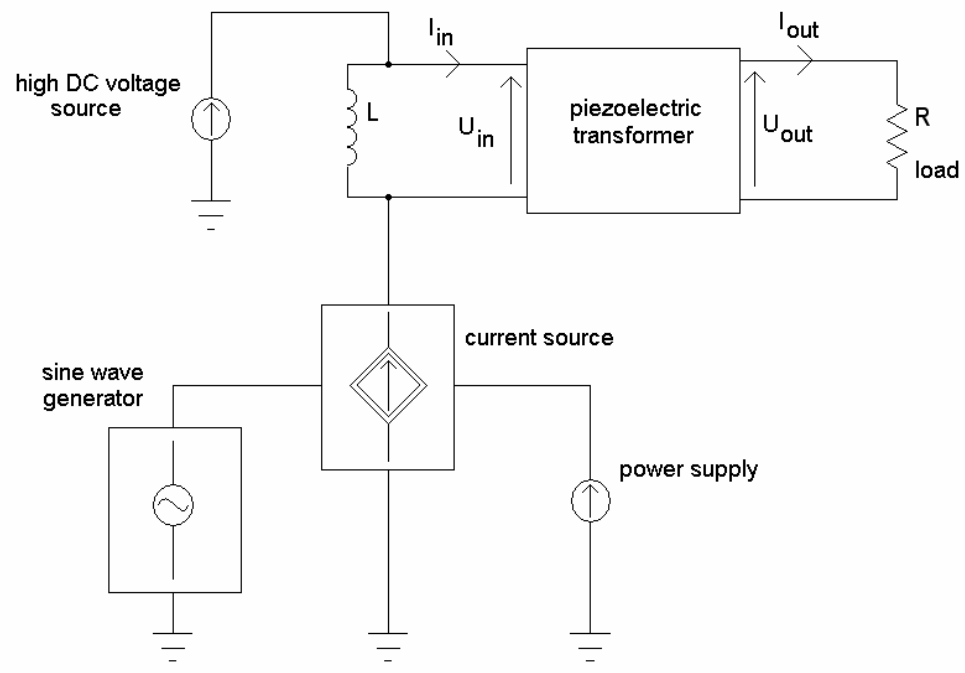

Fig. 5. General diagram of the test circuit for the efficiency measurement purposes.

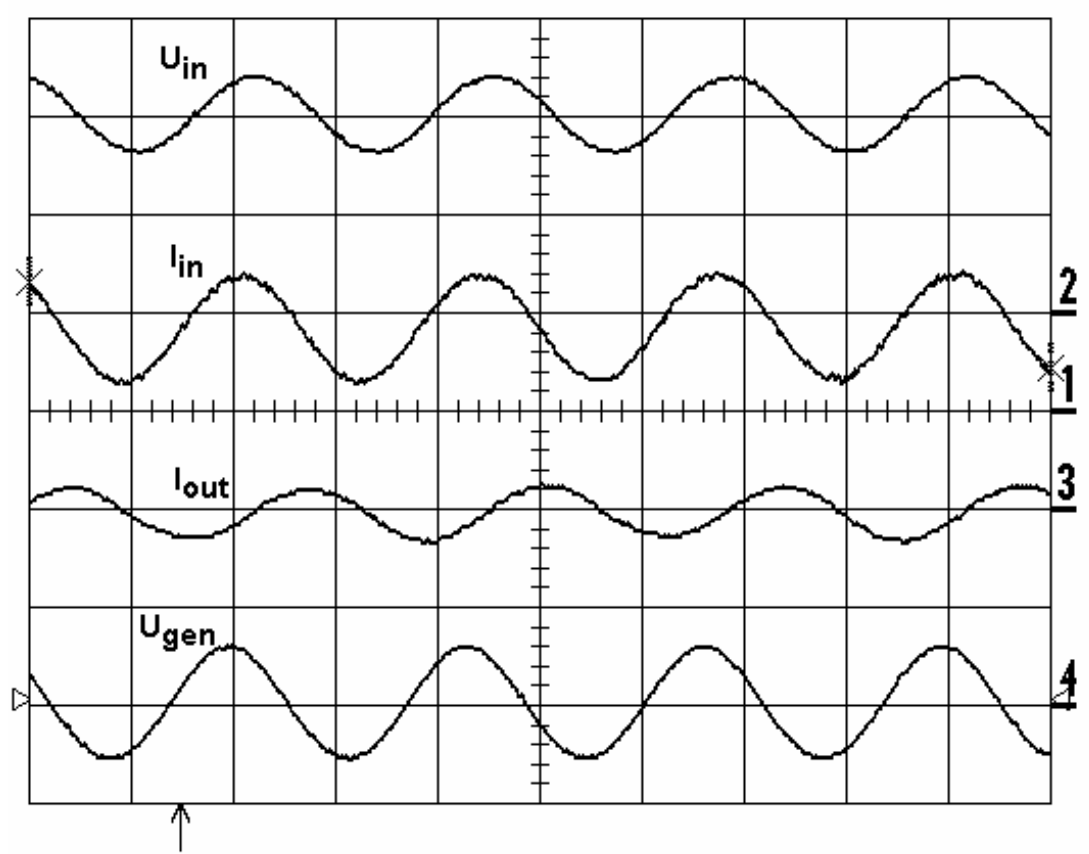

Fig. 6. Example waveforms at $f=430 \mathrm{kHz}$ and $P_{\text {out }}=0.4 \mathrm{~W}\left(U_{\text {in }}-\right.$ input voltage, $20 \mathrm{~V} / \mathrm{div}$; $l_{\text {in }}$ - input current, $200 \mathrm{~mA} /$ div; $I_{\text {out }}$ - output current, $1 \mathrm{~A} /$ div; $U_{\text {gen }}-$ generator output voltage, $5 \mathrm{~V} /$ div). 
http://dx.doi.org/10.21611/qirt.2006.093

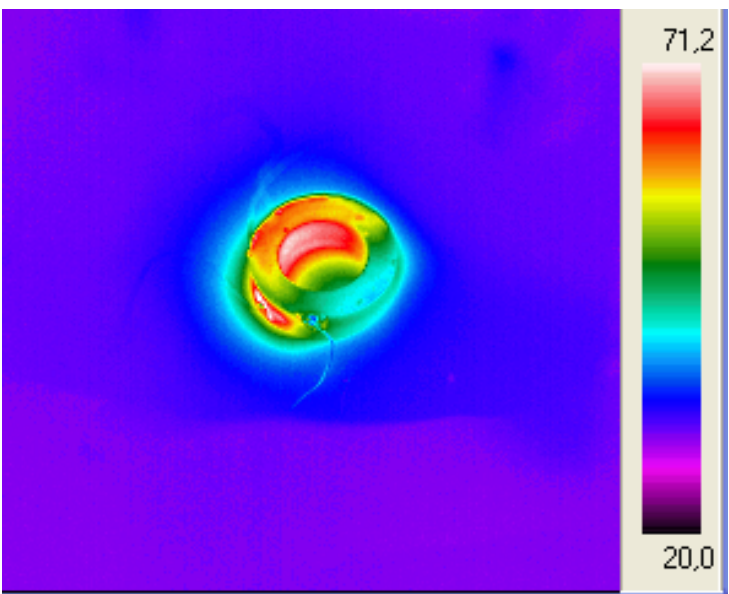

Fig. 7. Thermal image of the ring-shaped piezoelectric transformer acquired during one of the efficiency tests. 